\title{
ALMA Compact Array observations of the Fried Egg nebula
}

\section{Evidence for large-scale asymmetric mass-loss from the yellow hypergiant IRAS 17163-3907}

\author{
S. H. J. Wallström ${ }^{1}$, E. Lagadec ${ }^{2}$, S. Muller ${ }^{1}$, J. H. Black ${ }^{1}$, N. L. J. Cox ${ }^{3,4}$, R. Galván-Madrid ${ }^{5}$, K. Justtanont ${ }^{1}$, \\ S. Longmore ${ }^{6}$, H. Olofsson ${ }^{1}$, R. D. Oudmaijer ${ }^{7}$, G. Quintana-Lacaci ${ }^{8}$, R. Szczerba ${ }^{9}$, \\ W. Vlemmings ${ }^{1}$, H. van Winckel ${ }^{10}$, and A. Zijlstra ${ }^{11}$ \\ ${ }^{1}$ Department of Earth and Space Sciences, Chalmers University of Technology, Onsala Space Observatory, 439-92 Onsala, Sweden \\ e-mail: swallstrom@asiaa.sinica.edu.tw \\ 2 Laboratoire Lagrange, UMR 7293, Université Côte d'Azur, CNRS, Observatoire de la Côte d'Azur, Boulevard de l'Observatoire, \\ 06304 Nice Cedex 4, France \\ ${ }^{3}$ Université de Toulouse, UPS-OMP, IRAP, 31028 Toulouse, France \\ 4 CNRS, IRAP, 9 Av. colonel Roche, BP 44346, 31028 Toulouse, France \\ 5 Instituto de Radioastronomía y Astrofísica, UNAM, A.P. 3-72, Xangari, 58089 Morelia, Mexico \\ 6 Astrophysics Research Institute, Liverpool John Moores University, Liverpool L3 5RF, UK \\ 7 School of Physics and Astronomy, The University of Leeds, Leeds, LS2 9JT, UK \\ 8 Instituto de Ciencia de Materiales de Madrid, CSIC, C/Sor Juana Inés de la Cruz 3, 28049 Cantoblanco, Spain \\ 9 Nicolaus Copernicus Astronomical Center, Rabianska 8, 87-100 Torun, Poland \\ 10 Instituut voor Sterrenkunde, KU Leuven, Celestijnenlaan 200D, 3001 Heverlee, Belgium \\ 11 Jodrell Bank Centre for Astrophysics, Alan Turing Building, Manchester M13 9PL, UK
}

Received 1 March 2016 / Accepted 2 December 2016

\begin{abstract}
Yellow hypergiants are rare and represent a fast evolutionary stage of massive evolved stars. That evolutionary phase is characterised by a very intense mass loss, the understanding of which is still very limited. Here we report ALMA Compact Array observations of a 50"-mosaic toward the Fried Egg nebula, around one of the few Galactic yellow hypergiants IRAS 17163-3907. The emission from the ${ }^{12} \mathrm{CO} J=2-1$ line, $\mathrm{H} 30 \alpha$ recombination line, and continuum is imaged at a resolution of $\sim 8^{\prime \prime}$, revealing the morphology of the molecular environment around the star. The continuum emission is unresolved and peaks at the position of the star. The radio recombination line $\mathrm{H} 30 \alpha$ shows unresolved emission at the star, with an approximately Gaussian spectrum centered on a velocity of $21 \pm 3 \mathrm{~km} \mathrm{~s}^{-1}$ with a width of $57 \pm 6 \mathrm{~km} \mathrm{~s}^{-1}$. In contrast, the CO 2-1 emission is complex and decomposes into several components beyond the contamination from interstellar gas in the line of sight. The CO spectrum toward the star is a broad plateau, centered at the systemic velocity of $+18 \mathrm{~km} \mathrm{~s}^{-1}$ and with an expansion velocity of $100 \pm 10 \mathrm{~km} \mathrm{~s}^{-1}$. Assuming isotropic and constant mass-loss, we estimate a mass-loss rate of $8 \pm 1.5 \times 10^{-5} M_{\odot} \mathrm{yr}^{-1}$. At a radius of $25^{\prime \prime}$ from the star, we detect CO emission associated with the dust ring previously imaged by Herschel. The kinematics of this ring, however, is not consistent with an expanding shell, but show a velocity gradient of $v_{\text {sys }} \pm 20 \mathrm{~km} \mathrm{~s}^{-1}$. In addition, we find a puzzling bright feature radially connecting the star to the CO ring, at a velocity of $+40 \mathrm{~km} \mathrm{~s}^{-1}$ relative to the star. This spur feature may trace a unidirectional ejection event from the star. Our ACA observations reveal the complex morphology around IRAS 17163 and illustrate the breakthroughs that ALMA will bring to the field of massive stellar evolution.
\end{abstract}

Key words. circumstellar matter - stars: AGB and post-AGB - stars: mass-loss - stars: individual: IRAS 17163-3907

\section{Introduction}

Massive stars, with initial masses between 8 and $40 M_{\odot}$, spend only $\sim 10$ million years on the main sequence before becoming large and cool red supergiant (RSG) stars. The most massive of these stars, $M>20 M_{\odot}$, will then evolve via a short-lived $\left(10^{2-3} \mathrm{yr}\right)$ yellow hypergiant (YHG) phase, followed by a luminous blue variable phase, to finally become Wolf-Rayet stars (Oudmaijer et al. 2009). The intense mass-loss associated with these phases, and the eventual supernova explosion, provides kinetic energy and chemical enrichment to the surrounding interstellar medium (ISM). The stellar winds lead to the formation of a circumstellar envelope (CSE), that will affect the shape and evolution of the future supernova remnant, and determine how the material will be incorporated into the ISM. The CSE also traces the mass-loss history of the star, providing valuable information about the past evolution and possible future of these rare giants.

IRAS 17163-3907 (also called Hen 3-1379 or the Fried Egg nebula) is one of the brightest mid-infrared sources in the sky and was initially classified as a post-AGB star by Szczerba et al. (2007). More recently, the distance $(4 \pm 0.5 \mathrm{kpc})$ to this object has been well constrained by the presence of interstellar absorption lines in its optical spectra (Lagadec et al. 2011), implying that the star is four times further away than previously assumed. It is thus too luminous to be a post-AGB star. The location of IRAS 17163 on a temperature-luminosity diagram and the similarity of its optical spectrum with the 
well-studied YHG IRC+10420 (Wallström et al. 2015) suggest that its properties are very similar to the brightest YHGs (de Jager 1998). Hence, IRAS 17163 is one of the rare Galactic yellow hypergiants. These stars are known to have circumstellar envelopes of gas and dust, but only two other objects, IRC+10420 and AFGL 2343, have an extended molecular envelope (Castro-Carrizo et al. 2007). Both objects show an overall spherical symmetry, and evidence of a detached shell.

Resolved images of IRAS 17163 and its envelope (Lagadec et al. 2011), obtained using the VLT mid-infrared instrument VISIR, allowed the dust distribution around the central star to be mapped. Two concentric spherical dusty shells were resolved within $2.5^{\prime \prime}$ of the star (note that at a distance of $4 \mathrm{kpc}, 1^{\prime \prime}$ corresponds to $\sim 0.02 \mathrm{pc}$ or $\left.4000 \mathrm{AU}\right)$. Radiative transfer modeling of the circumstellar environment indicates the presence of $0.04 M_{\odot}$ of dust in the envelope, ejected during the last few hundred years. Furthermore, a larger, cooler dust shell with a radius $\sim 25^{\prime \prime}$ was discovered with the Herschel Space Observatory (Hutsemékers et al. 2013) and they estimate the total mass of gas and dust, including this shell, to be $\sim 7 M_{\odot}$ (assuming a gas-to-dust mass ratio of 40).

To further constrain the properties of the circumstellar envelope of IRAS 17163, particularly the mass-loss rate and kinematics, Wallström et al. (2015) performed spectral observations of the CO $J=2-1$ and 3-2 lines with the Atacama Pathfinder Experiment (APEX) telescope. They find a complex CO spectrum with asymmetric line profiles and multiple velocity components in addition to the contamination by interstellar gas in the line of sight. However, the limited spatial resolution and sensitivity prevented them from fully interpreting the complex emission.

We have obtained continuum and CO 2-1 Atacama Large Millimeter/Submillimeter Array (ALMA) compact array (ACA) observations that allow us to shed light on the morphology and kinematics of the circumstellar environment of IRAS 17163. These observations are presented in Sect. 2, followed by Results in Sect. 3, a Discussion in Sect. 4, and finally Conclusions in Sect. 5.

\section{Observations and data reduction}

\subsection{ALMA Compact Array data}

IRAS 17163 was observed as part of project 2013.1.00502.S with the ALMA Compact Array (ACA) between the 25th and 27 th of July 2014, in a mosaic of seven pointings to cover a field of $\sim 50^{\prime \prime}$. The mosaic was centered on RA 17:19:49.335 and Dec $-39: 10: 37.94$ (J2000), with the six additional pointings offset by $\sim 28^{\prime \prime}$ in a hexagonal pattern. The array configuration and $u v$-coverage resulted in a synthesized beam of $8.4^{\prime \prime} \times 4.3^{\prime \prime}$ (with a position angle of $96.5^{\circ}$ ) at the frequency of the $\mathrm{CO} 2-$ 1 line, using natural weighting. The projected baselines ranged between $\sim 5$ and $45 \mathrm{~m}$, giving a maximum recoverable scale of $\sim 30^{\prime \prime}$.

Two spectral windows $1.875 \mathrm{GHz}$ wide were set up with $0.977 \mathrm{MHz}$ channel spacing, centered at $230.481 \mathrm{GHz}$ (upper side band, covering the $\mathrm{CO} 2-1$ transition) and $217.047 \mathrm{GHz}$ (lower side band, covering the $\mathrm{SiO} 5-4$ transition, which was not detected). The velocity resolution for these windows is $1.7 \mathrm{~km} \mathrm{~s}^{-1}$ after Hanning smoothing. In addition, two more 2-GHz windows were set up with a coarse spectral resolution of $15.6 \mathrm{MHz}$ for continnum imaging, centered at frequencies of 215.9 and $231.5 \mathrm{GHz}$. We detect emission from the $\mathrm{H} 30 \alpha$ line in the latter.
Data were calibrated and imaged in a standard way using $\mathrm{CASA}^{1}$. The Jy-to-K conversion factor to brightness temperature for the CO 2-1 data is $1 \mathrm{Jy}=0.637 \mathrm{~K}$. Continuum was subtracted from the line emission data by using the CASA task uvcontsub and fitting a first order polynomial to line-free channels.

\subsection{APEX data}

Complementary APEX ${ }^{2}$ observations were carried out with the APEX-1 receiver (Vassilev et al. 2008) on August 27th, 2015 under very good weather conditions (precipitable water vapor of $\sim 0.8 \mathrm{~mm}$ ). The ${ }^{13} \mathrm{CO} 2-1$ transition at $220.4 \mathrm{GHz}$ was observed in a single pointing centered on IRAS 17163 (main beam 28"), with the same fixed off-position used by Wallström et al. (2015), $\sim 40^{\prime \prime}$ south-west of the star. The data were reduced in the standard single-dish data reduction package CLASS ${ }^{3}$.

\section{Results}

In this section, we describe the morphology of the $\mathrm{CO} 2-1$ emission revealed by the ACA. We identify several distinct features in the data, which is shown smoothed to the resolution of APEX in Fig. 1a. Between -50 and $0 \mathrm{~km} \mathrm{~s}^{-1}$ we see widespread ISM emission, around the systemic velocity at $+18 \mathrm{~km} \mathrm{~s}^{-1}$ (see Sect. 3.3) is emission from a clumpy CO ring, between +50 and $+80 \mathrm{~km} \mathrm{~s}^{-1}$ is a bright feature we call the "spur", and between 90 and $110 \mathrm{~km} \mathrm{~s}^{-1}$ is emission from a western arc. Each of these components is discussed in a separate section below.

In addition, we find the $\mathrm{H} 30 \alpha$ recombination line of hydrogen at $231.9 \mathrm{GHz}$ as a spatially unresolved feature centered on IRAS 17163. The line profile is resolved with a velocity resolution of $\sim 20 \mathrm{~km} \mathrm{~s}^{-1}$, and a Gaussian fit finds the line centered on $21 \pm 3 \mathrm{~km} \mathrm{~s}^{-1}$ with a FWHM of $57 \pm 6 \mathrm{~km} \mathrm{~s}^{-1}$ and a peak brightness temperature of $\sim 9 \mathrm{mK}$ (Fig. 1c).

Figure 2 presents integrated maps over various features. The dashed circle represents the approximate location of the "Herschel ring", a cool dust ring seen with Herschel (Hutsemékers et al. 2013), with a radius of 25" and centered on IRAS 17163. All velocities are given in the local standard restframe (LSR). Channel maps between -100 and $+150 \mathrm{~km} \mathrm{~s}^{-1}$ are also presented in Fig. A.1.

\subsection{Continuum emission}

The continuum emission (Fig. 3), obtained by aggregating all line-free channels in the coarse spectral resolution windows, is an unresolved feature at the location of the star, with flux densities of 42 and $48 \mathrm{mJy}$ in the two bands centered on 215.9 and $231.5 \mathrm{GHz}$, respectively. SED fitting by Hutsemékers et al. (2013) found two dust components at temperatures of $\sim 180$ and $60 \mathrm{~K}$. However, their longest wavelength observations are $160 \mu \mathrm{m}(\simeq 1880 \mathrm{GHz})$, so the cold dust is not well constrained. Extrapolating their SED fit (modified blackbody emission with $\beta=2$ ) gives fluxes $<20 \mathrm{mJy}$ around $230 \mathrm{GHz}$. We find values a factor of $\sim 2$ larger, suggesting there may be a colder dust component within $8^{\prime \prime}$ of the star.

\footnotetext{
1 http://casa.nrao.edu/

2 This publication is based on data acquired with the Atacama Pathfinder Experiment (APEX). APEX is a collaboration between the Max-Planck-Institut für Radioastronomie, the European Southern Observatory, and the Onsala Space Observatory.

3 http://www . iram. fr/IRAMFR/GILDAS
} 


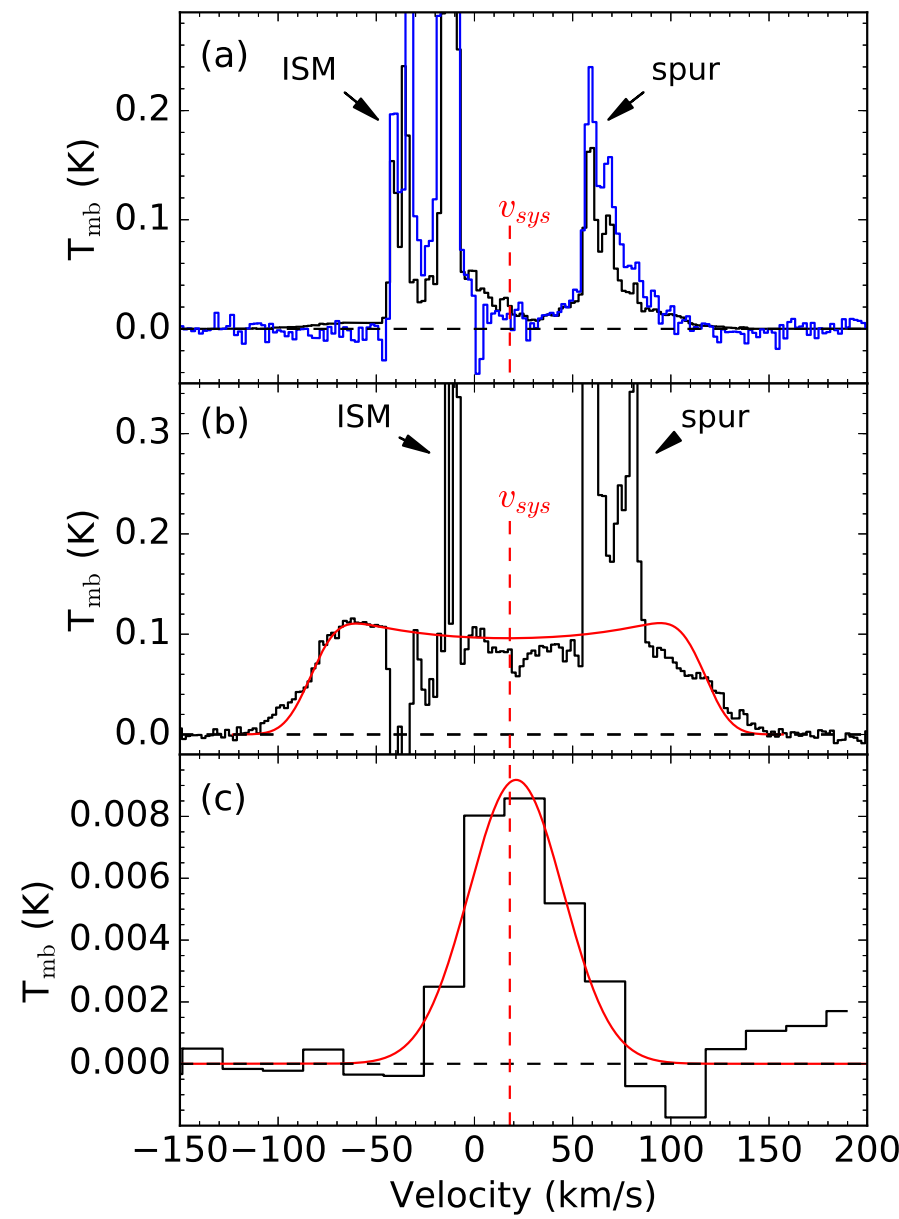

Fig. 1. a) In black, a spectrum of the CO 2-1 ACA data smoothed to the 27" beam of APEX. In blue, the APEX CO 2-1 spectrum from Wallström et al. (2015). b) ACA CO 2-1 spectrum summed over the synthesised beam at the position of IRAS 17163, showing the broad plateau seen only towards the star. The best fit mass-loss model is overlaid in red. c) The $\mathrm{H} 30 \alpha$ spectrum, averaged over the synthesised beam at the position of IRAS 17163. The best fit Gaussian line profile is overlaid in red, centered on $21 \mathrm{~km} \mathrm{~s}^{-1}$ with a FWHM of $57 \mathrm{~km} \mathrm{~s}^{-1}$. In all spectra the systemic velocity at $+18 \mathrm{~km} \mathrm{~s}^{-1}$ (see Sect. 3.3) is marked with a dashed red line, and in spectra a) and b) the absorption-like features between -50 and $0 \mathrm{~km} \mathrm{~s}^{-1}$ are artefacts caused by the filtering out of extended emission.

\subsection{The interstellar surroundings}

IRAS 17163 is in a complex region of the sky, with widespread CO (Arnal et al. 2008) and cool dust (Hutsemékers et al. 2013) emission. Indeed, the brightest $\mathrm{CO}$ emission in the ACA dataset is ISM emission which covers the field of view between roughly -50 and $0 \mathrm{~km} \mathrm{~s}^{-1}$, as seen in Figs. 1a and 2c. The contamination from these ISM features precludes the analysis of any potential circumstellar emission at velocities in this range. There are two distinct ISM components, centered around -38 and $-12 \mathrm{~km} \mathrm{~s}^{-1}$, which match the CO 1-0 emission seen towards the nearby star forming regions RCW 121 and RCW 122 (Arnal et al. 2008). These velocities are consistent with Galactic rotation in the direction of IRAS 17163 at distances of 4 and $1 \mathrm{kpc}$, respectively (Reid et al. 2009). In addition, we note a fainter diffuse filament around $-150 \mathrm{~km} \mathrm{~s}^{-1}$ (see Fig. 2a), which is consistent with Galactic rotation at $7 \mathrm{kpc}$.

We note that our mosaic does not cover the full extent of the widespread ISM emission around -38 and $-12 \mathrm{~km} \mathrm{~s}^{-1}$. This causes negative artefacts in the data, due to the filtering out of extended emission $\left(>30^{\prime \prime}\right)$ by the interferometric observations.

\subsection{The stellar wind}

Figure 1b shows the CO 2-1 spectrum extracted toward IRAS 17163. It was obtained by summing the emission within the synthesized beam centered on the star. In this spectrum, the most prominent emission feature $\left(v \sim-10 \mathrm{~km} \mathrm{~s}^{-1}\right)$ corresponds to interstellar $\mathrm{CO}$, as discussed above. Next is the bright feature between $\sim 50$ and $80 \mathrm{~km} \mathrm{~s}^{-1}$ that was observed in previous APEX spectra (Wallström et al. 2015). We discuss this feature, which we call the spur, hereafter in Sect. 3.5. Finally, clearly detected with the ACA, is a faint $(\sim 0.1 \mathrm{~K}$ in the ACA beam) plateau centered on a velocity of $\sim 20 \mathrm{~km} \mathrm{~s}^{-1}$ and with a broad width of $\sim 250 \mathrm{~km} \mathrm{~s}^{-1}$, seen only towards the star (see Figs. $2 \mathrm{~b}$, $\mathrm{d}, \mathrm{f}$ ). This resolves the previously found velocity discrepancy between APEX CO peak emission around $+60 \mathrm{~km} \mathrm{~s}^{-1}$ and optical Fe II emission lines at $+18 \mathrm{~km} \mathrm{~s}^{-1}$ (Wallström et al. 2015). Henceforth we take the systemic velocity of IRAS 17163 to be $+18 \mathrm{~km} \mathrm{~s}^{-1}$.

Assuming the plateau corresponds to a stellar wind, we use a simple model of an expanding circumstellar envelope where the radiative transfer is solved using a Monte Carlo method (Bernes 1979; Schöier \& Olofsson 2001) to constrain the mass-loss rate. This model assumes that the envelope expands at a constant velocity and is formed by a constant, isotropic, and smooth massloss. It incorporates CO rotational energy levels up to $J=40$ for the vibrational ground state and the first vibrationally excited state, and uses collisional cross-sections from Yang et al. (2010). In our modeling we take the stellar parameters (distance, effective temperature, luminosity) from Lagadec et al. (2011), and assume a $[\mathrm{CO}] /\left[\mathrm{H}_{2}\right]$ ratio of $10^{-4}$, a turbulent velocity of $1 \mathrm{~km} \mathrm{~s}^{-1}$, and a gas-to-dust ratio of 40 (cf. Hutsemékers et al. 2013). For our parameters the model finds a $\mathrm{CO}$ half-abundance radius (determined by photodissociation) of $1.55 \times 10^{17} \mathrm{~cm}$, corresponding to $2.6^{\prime \prime}$ at $4 \mathrm{kpc}$, consistent with the extent of the dust shells found by Lagadec et al. (2011).

In order to fit the mass-loss rate and expansion velocity, we executed a grid of models over the parameter space and determined the best fit model by $\chi^{2}$ minimisation to the parts of the spectrum not contaminated with ISM or spur emission. We find an expansion velocity of $100 \pm 10 \mathrm{~km} \mathrm{~s}^{-1}$ and a mass-loss rate of $8 \pm 1.5 \times 10^{-5} M_{\odot} \mathrm{yr}^{-1}$ (Fig. 1b). These errors are the statistical errors corresponding to the $1 \sigma \chi^{2}$ error ellipse. The mass-loss rate estimate has further uncertainty associated with the model parameters, such as the distance to the star and the assumption of constant and isotropic mass-loss, which are not taken into account here. Integrating over the model fit we find a total integrated intensity of $\sim 21 \mathrm{~K} \mathrm{~km} \mathrm{~s}^{-1}$ for the wind.

We note that the model was developed for the circumstellar envelopes of AGB stars, and hence may not be strictly applicable to our YHG star. For example, it assumes an entirely molecular gas envelope, whereas the envelope of IRAS 17163 might be significantly atomic due to the high stellar temperature. On the other hand, this method provides an order of magnitude estimate of the mass-loss rate, allowing us to make comparisons to other massive evolved stars. Accordingly, our estimated mass-loss rate is similar to that of the archetypal YHG IRC +10420 , of $\sim 1-9 \times$ $10^{-4} M_{\odot} \mathrm{yr}^{-1}$ (Castro-Carrizo et al. 2007; Dinh-V.-Trung et al. 2009).

From the expansion velocity, $\mathrm{CO}$ half-abundance radius, and (assumed constant) mass-loss rate we can estimate a total gas mass for this stellar wind of $0.04 M_{\odot}$. We note that this estimate 

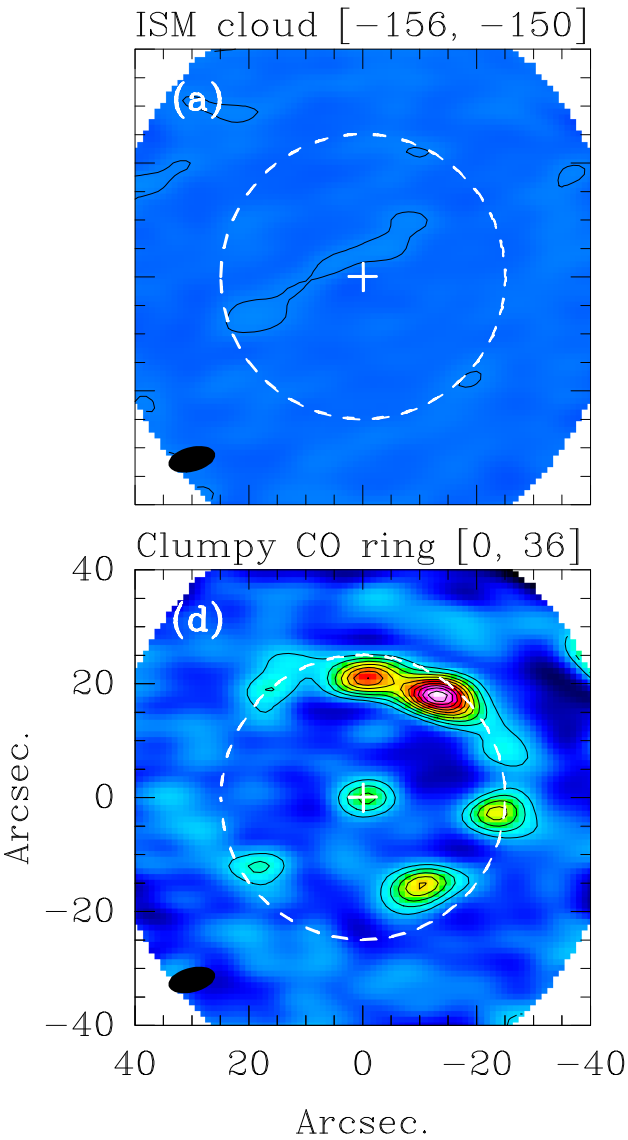
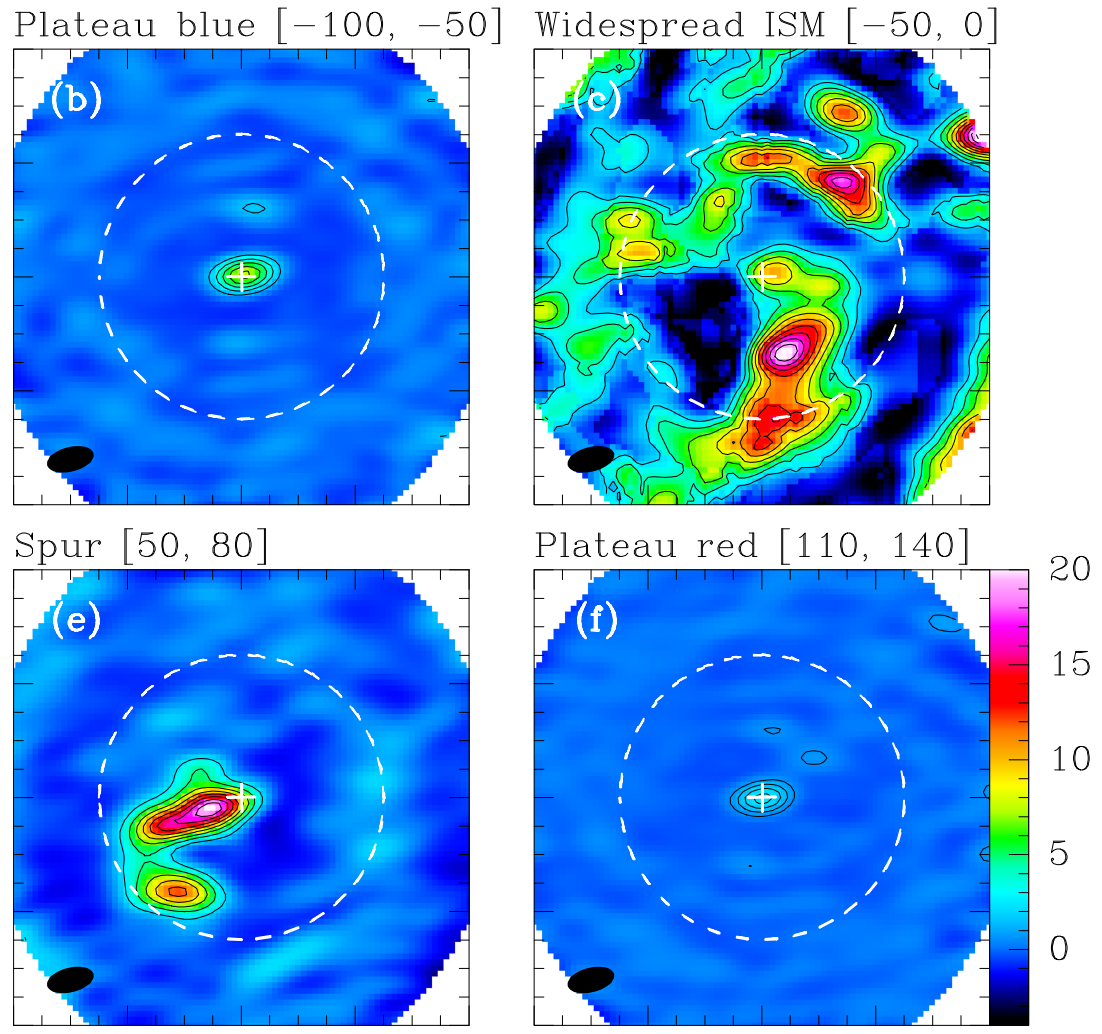

Fig. 2. Integrated CO 2-1 maps over several different features in the ACA data, with the velocity range of each map given in brackets. North is up and East is to the left in each map. a) An interstellar cloud around $-150 \mathrm{~km} \mathrm{~s}^{-1}$. Contours start at $4 \sigma$ and are spaced by $4 \sigma\left(\sigma=57 \mathrm{mK} \mathrm{km} \mathrm{s}{ }^{-1}\right)$. b) The blue edge of the stellar wind plateau between -100 and $-50 \mathrm{~km} \mathrm{~s}^{-1}$. Contours start at $10 \sigma$ and are spaced by $10 \sigma(\sigma=146 \mathrm{mK} \mathrm{km} \mathrm{s})$. c) Widespread ISM emission between -50 and $0 \mathrm{~km} \mathrm{~s}^{-1}$. Contours start at $15 \sigma$ and are spaced by $15 \sigma\left(\sigma=140 \mathrm{mK} \mathrm{km} \mathrm{s}{ }^{-1}\right)$. d) The stellar wind at the star, and the clumpy $\mathrm{CO}$ ring, integrated between 0 and $+36 \mathrm{~km} \mathrm{~s}^{-1}$, symmetrically around the systemic velocity. Contours start at $15 \sigma$ and are spaced by $15 \sigma\left(\sigma=124 \mathrm{mK} \mathrm{km} \mathrm{s}^{-1}\right)$. e) The spur, integrated between 50 and $80 \mathrm{~km} \mathrm{~s}^{-1}$. Contours start at $20 \sigma$ and are spaced by $20 \sigma$ $\left(\sigma=114 \mathrm{mK} \mathrm{km} \mathrm{s}^{-1}\right)$. f) The red edge of the stellar wind plateau between +110 and $+140 \mathrm{~km} \mathrm{~s}^{-1}$. Contours start at $5 \sigma$ and are spaced by $5 \sigma$ $\left(\sigma=114 \mathrm{mK} \mathrm{km} \mathrm{s}^{-1}\right)$. The restoring beam $\left(8.4^{\prime \prime} \times 4.3^{\prime \prime}\right)$ is shown as a filled ellipse in the bottom left of each map, and the common color scale is shown in the bottom right map. The location of the star is marked with a cross and the dust shell seen with Herschel is represented by a dashed circle with $25^{\prime \prime}$ radius.

assumes an entirely molecular wind and hence, as there may be additional mass outside the extent of the $\mathrm{CO}$ envelope, it should be viewed as a lower bound on the total gas mass. Our estimate is similar to the mass found in the inner $2.5^{\prime \prime}$ region by Hutsemékers et al. (2013) of $0.08 M_{\odot}$, assuming a gas-to-dust ratio of 40. On the other hand, Lagadec et al. (2011) find a much larger mass in this region: a total dust mass of $0.04 M_{\odot}$, the bulk of which is in the inter-shell region between two concentric dust shells. Note, however, that the inter-shell dust was added, and the outer radius in the Lagadec et al. (2011) model was arbitrary extended to about $5^{\prime \prime}$, in an attempt to explain the IRAS fluxes which we now know are mostly due to the emission from the Herschel dust shell (Hutsemékers et al. 2013). Hence, updating the Lagadec et al. (2011) modelling of the inner shells, without the IRAS fluxes and with better constraints on the radial boundaries, results in a total dust mass of $0.003 M_{\odot}$ which, assuming a gas-to-dust ratio of 40 , corresponds to a gas mass of $0.12 M_{\odot}$. Given the uncertainties in the models of both gas and dust, this is in reasonable agreement with our lower bound estimate of $0.04 M_{\odot}$.

The expansion velocity of $100 \pm 10 \mathrm{~km} \mathrm{~s}^{-1}$ is unusually high for a post-RSG star: Oudmaijer et al. (2009) found typical velocities of $\sim 20 \mathrm{~km} \mathrm{~s}^{-1}$. They found only two objects with similar outflow velocities, the warm supergiants HD 101584 and Frosty Leo, both of which have bipolar outflows. Some OH/IR stars with bipolar outflows also show similarly large expansion velocities (Zijlstra et al. 2001). Although it is not known what powers such high velocity outflows, they seem more likely to be associated with a bipolar flow. However, the central emission associated with the fast stellar wind of IRAS 17163 is unresolved in the ACA beam so its geometry remains elusive.

\subsection{The clumpy CO ring}

In addition to the central unresolved emission from the stellar wind, the CO channel maps show a series of clumps whose overall distribution reproduces the cool $(T \sim 60 \mathrm{~K})$ dust ring seen with Herschel (Hutsemékers et al. 2013; see Fig. 4). These clumps have a wide range of velocities but the contamination with interstellar $\mathrm{CO}$ (at $v<0 \mathrm{~km} \mathrm{~s}^{-1}$ ) on one side and the spur $\left(v \sim 50-80 \mathrm{~km} \mathrm{~s}^{-1}\right)$ on the other makes it difficult to define the total extent in velocity. In any case, the overall kinematics clearly do not reflect that of an expanding shell. A shell expanding at constant velocity would show up in the channel maps (Fig. A.1) projected as a ring changing in size with velocity and reaching 


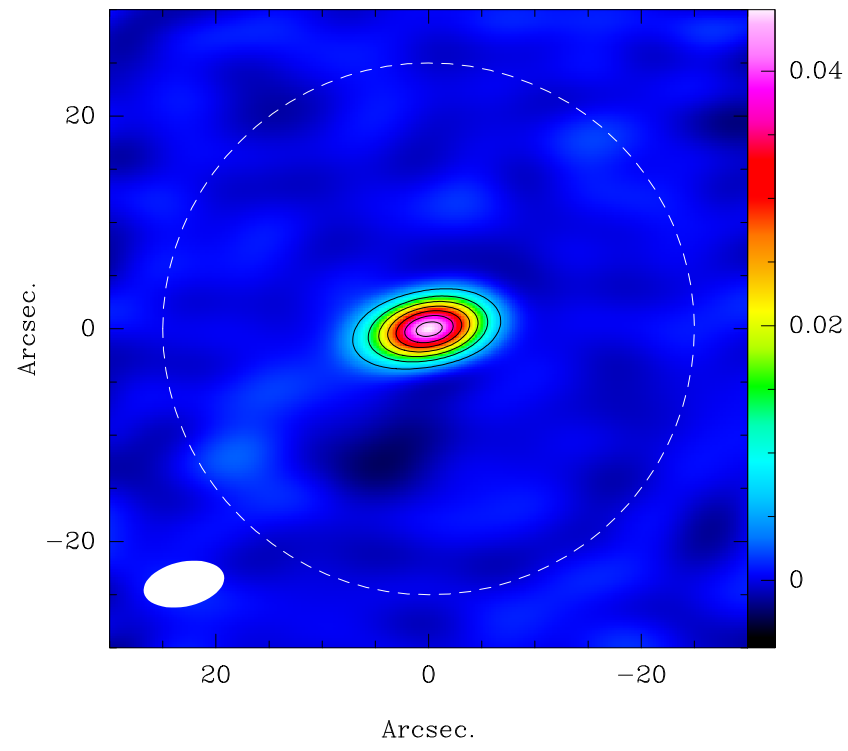

Fig. 3. The ACA continuum emission, showing the central unresolved feature. Contours start at $10 \sigma(\sigma=0.6 \mathrm{mJy} / \mathrm{beam})$ and are spaced by $10 \sigma$. The location of the Herschel dust shell is shown with a dashed $25^{\prime \prime}$-radius circle, and the restoring beam is shown as a white ellipse in the bottom left.

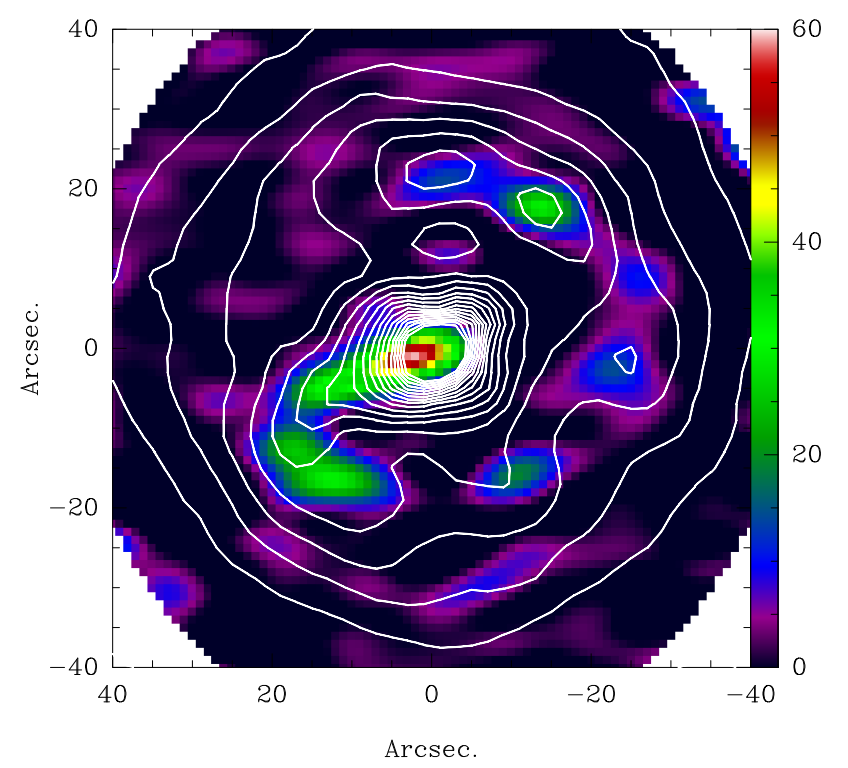

Fig. 4. In color: ACA CO 2-1 emission integrated over the velocity range 0 to $+140 \mathrm{~km} \mathrm{~s}^{-1}$ (avoiding the ISM contamination). Note that the emission peaks not at the star but on the spur feature. Overlaid in gray are Herschel $70 \mu \mathrm{m}$ dust contours (see Hutsemékers et al. 2013).

a maximum extent at the systemic velocity. Instead, the velocity structure of the clumpy $\mathrm{CO}$ ring shows a strong velocity gradient with red-shifted clumps to the south-east and blue-shifted clumps to the north-west (Fig. 5). Note that Fig. 5 shows a restricted velocity range of $\pm 20 \mathrm{~km} \mathrm{~s}^{-1}$ around the systemic velocity to avoid confusion from the ISM or spur features. The integrated intensity of this clumpy ring, between 0 and $36 \mathrm{~km} \mathrm{~s}^{-1}$, is $\sim 65 \mathrm{~K} \mathrm{~km} \mathrm{~s}^{-1}$.

In order to estimate the masses of the CO clumps, we used the radiative transfer code RADEX (van der Tak et al. 2007) to find the range of parameters needed to reproduce the average peak clump brightness in CO 2-1, of $1.1 \pm 0.7 \mathrm{~K}$. To this end we fixed the linewidth to $10 \mathrm{~km} \mathrm{~s}^{-1}$ and the background temperature

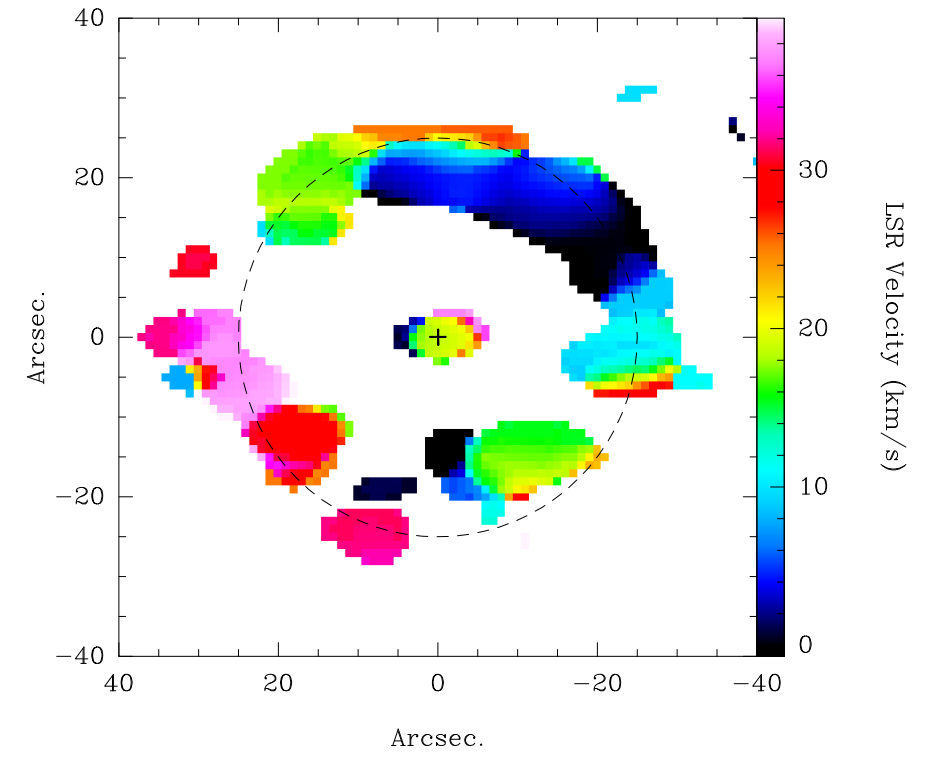

Fig. 5. An intensity weighted mean velocity map of the clumpy CO ring integrated within $20 \mathrm{~km} \mathrm{~s}^{-1}$ of the systemic velocity at $+18 \mathrm{~km} \mathrm{~s}^{-1}$, i.e. between -2 and $+38 \mathrm{~km} \mathrm{~s}^{-1}$, to avoid any confusion from the ISM or spur features. The location of the star is marked with a cross, and the location of the Herschel dust ring is indicated with a dashed circle of $25^{\prime \prime}$ radius.

to $2.73 \mathrm{~K}$, and explored a large parameter space over kinetic temperature, $\mathrm{H}_{2}$ density, and $\mathrm{CO}$ column density. The temperature and $\mathrm{H}_{2}$ density could not be constrained, but the $\mathrm{CO}$ column density was found to be $10^{16-17} \mathrm{~cm}^{-2}$, which corresponds to a mass of $0.03-0.3 M_{\odot}$ per clump (assuming a spherical clump diameter of $8^{\prime \prime}$ and a $\mathrm{CO}$ abundance of $10^{-4}$ compared with $\mathrm{H}_{2}$ ). We identify at least 8 separate clumps and hence a minimum total mass in the ring of $\sim 0.2-2 M_{\odot}$.

\subsection{The spur}

In the velocity range $\sim+50$ to $+80 \mathrm{~km} \mathrm{~s}^{-1}$ (LSR) there is a bright elongated $\mathrm{CO}$ feature we call the spur, extending radially from IRAS 17163 towards the south-east (position angle of $112^{\circ}$ ) to the clumpy $\mathrm{CO}$ ring. It is redshifted with respect to the star by $\sim 30-60 \mathrm{~km} \mathrm{~s}^{-1}$. This component corresponds to the emission seen in the APEX spectra by Wallström et al. (2015) and reaches a peak brightness temperature of $7.8 \mathrm{~K}$ in the ACA beam. The integrated intensity, between 50 and $80 \mathrm{~km} \mathrm{~s}^{-1}$, is $\sim 83 \mathrm{~K} \mathrm{~km} \mathrm{~s}^{-1}$. The spectrum of the spur shows a bright peak at $+58 \mathrm{~km} \mathrm{~s}^{-1}$ and fainter peaks at +66 and $+80 \mathrm{~km} \mathrm{~s}^{-1}$. The spatial structure of the spur is complex, seeming to break up into several components between +65 and $+80 \mathrm{~km} \mathrm{~s}^{-1}$ (see Fig. A.1). We also note that the spur appears in the Herschel dust contours (Fig. 4), suggesting it contains dust at a temperature of $60 \mathrm{~K}$. To obtain a rough estimate of the mass of the spur we apply the same method as described in Sect. 3.4 to find $N_{\mathrm{CO}}=10^{18} \mathrm{~cm}^{-2}$, corresponding to a gas mass of $\sim 3 M_{\odot}$ for an ellipse with major and minor axes of $\sim 15^{\prime \prime}$ and $5^{\prime \prime}$, respectively.

One way to determine if the spur is associated with the star is to measure the ${ }^{12} \mathrm{C} /{ }^{13} \mathrm{C}$ ratio. We obtained an APEX spectrum in ${ }^{13} \mathrm{CO} 2-1$ (Fig. 6) and compared with the ${ }^{12} \mathrm{CO}$ spectrum from Wallström et al. (2015). The ${ }^{13} \mathrm{CO}$ spectrum shows peaks coincident with the ${ }^{12} \mathrm{CO}$ peaks, but it is very faint with a peak brightness of $0.02 \mathrm{~K}$, corresponding to a signal-to-noise ratio of 4 . In order to estimate the ${ }^{12} \mathrm{CO} /{ }^{13} \mathrm{CO}$ ratio we fit both 


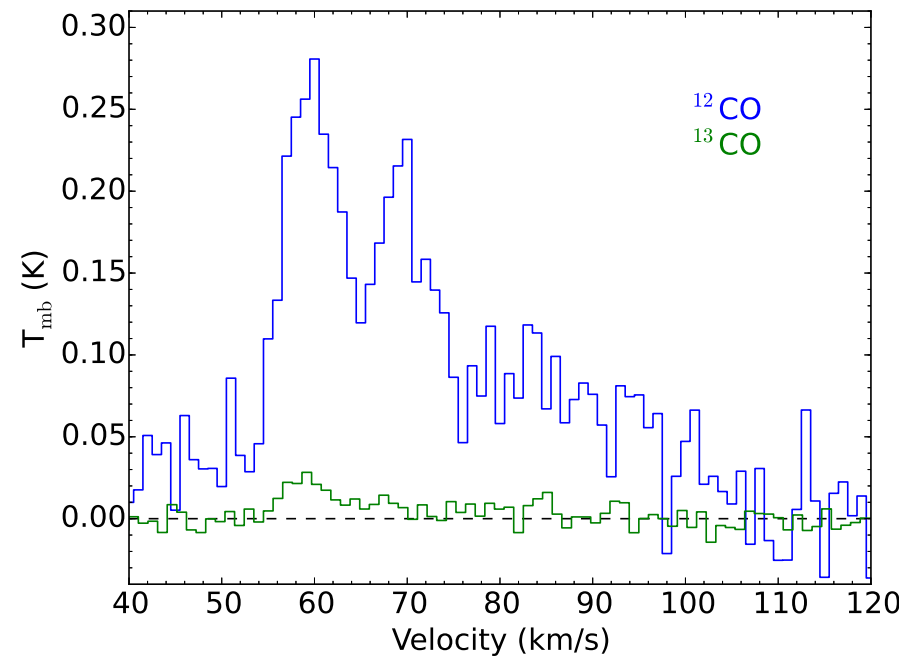

Fig. 6. APEX observations of ${ }^{12} \mathrm{CO}$ (blue) and ${ }^{13} \mathrm{CO}$ (green) $2-1$ emission around IRAS 17163.

spectra simultaneously with the same profile, multiplied by a scaling factor. The profile was well decomposed into three Gaussian components. We find a ${ }^{12} \mathrm{CO} /{ }^{13} \mathrm{CO}$ intensity ratio of $13_{-5}^{+10}$. Equating this to the ${ }^{12} \mathrm{C} /{ }^{13} \mathrm{C}$ abundance ratio requires the assumption that ${ }^{12} \mathrm{CO}$ is optically thin. This is likely, given that the spur is resolved in the ACA data and has a peak temperature of $\sim 8 \mathrm{~K}$, far below the $60 \mathrm{~K}$ temperature of the associated dust. $\mathrm{A}{ }^{12} \mathrm{C} /{ }^{13} \mathrm{C}$ abundance ratio around 10 is indicative of material ejected from an RSG star (Gonzalez \& Wallerstein 2000; Origlia et al. 2013), while the ISM ratio at the galactic radius of IRAS 17163 is around 40 (Wilson \& Rood 1994; assuming a distance of $4 \mathrm{kpc})$. Hence, this material was most likely ejected by the star in its previous RSG phase.

\subsection{Western arc}

Between +90 and $+110 \mathrm{~km} \mathrm{~s}^{-1}$ there is a western arc feature, which is not spatially connected with the spur emission. It is also not coincident with the clumpy $\mathrm{CO}$ ring (see Fig. 7). This feature is redshifted by $\sim 70-90 \mathrm{~km} \mathrm{~s}^{-1}$ with respect to the systemic velocity of the star, and has no blueshifted counterpart. Its velocity is also far removed from Galactic rotation at any distance towards IRAS 17163, suggesting it is not interstellar but instead connected with the star. However, the origin and nature of this western arc is unknown.

The western arc has an integrated intensity of $\sim 8 \mathrm{~K} \mathrm{~km} \mathrm{~s}^{-1}$ between 90 and $110 \mathrm{~km} \mathrm{~s}^{-1}$. Using the method from Sect. 3.4 to roughly estimate the mass of this feature, we find $N_{\mathrm{CO}}=$ $10^{16} \mathrm{~cm}^{-2}$, and, assuming spherical clumps of size $8^{\prime \prime}$, estimate a total mass of $\sim 0.09 M_{\odot}$ in three clumps.

\section{Discussion}

The ACA data reveal for the first time the distribution of the molecular gas in the Fried Egg nebula and allow us to reconcile the previous APEX spectra with optical and Herschel data. They also reveal new features, like the kinematics of the clumpy $\mathrm{CO}$ ring and the strong asymmetry of the spur structure, that call for further investigation.

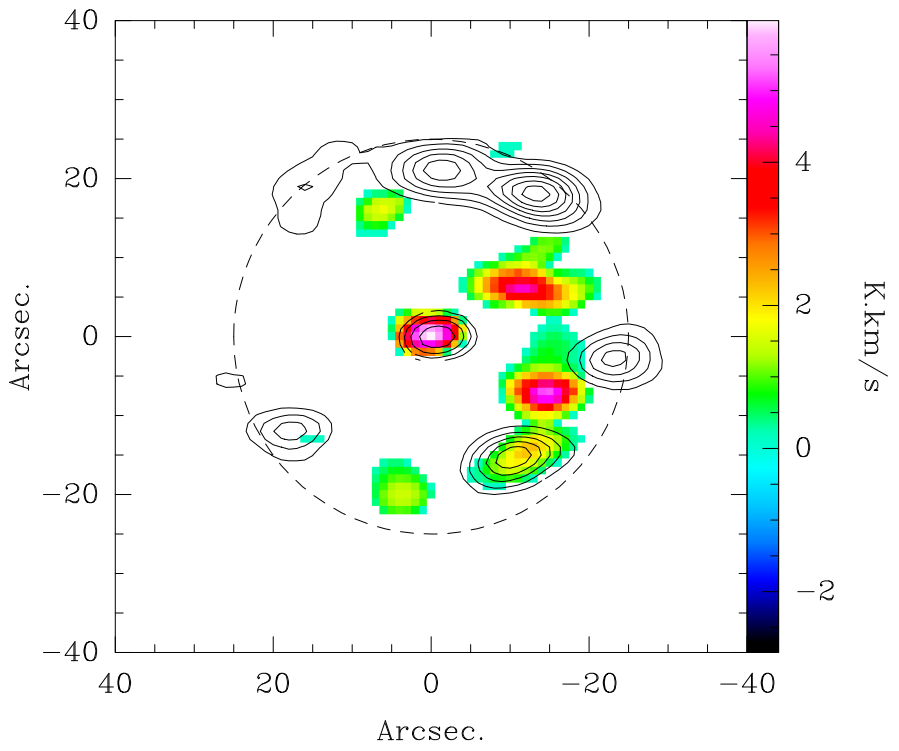

Fig. 7. In color: integrated CO $2-1$ map between +90 and $+110 \mathrm{~km} \mathrm{~s}^{-1}$, showing the western arc. The contours are taken from Fig. $2 \mathrm{~d}$ and show the clumpy CO ring, and the dust shell seen with Herschel is represented by a dashed circle with $25^{\prime \prime}$ radius. As can be seen, the brightest arc emission is not coincident with the $\mathrm{CO}$ ring.

\subsection{Origin of the clumpy CO ring}

The clumpy CO ring is formed by a collection of apparently individual clumps which collectively have (i) a coherent kinematic structure; and (ii) an roughly circular symmetry with respect to the star; which (iii) spatially matches the $\sim 60 \mathrm{~K}$ dust emission seen with Herschel. The symmetry strongly implies an origin connected to the star and, as discussed in Sect. 3.4, the kinematics are not compatible with an expanding shell. From the velocity structure the ring instead resembles a torus, close to the plane of the sky (inclination $\sim 35^{\circ}$, assuming circular symmetry). From the velocities of the clumps we estimate the ejection velocity is between 20 and $\sim 50 \mathrm{~km} \mathrm{~s}^{-1}$, meaning the torus was ejected between $\sim 7000$ and $20000 \mathrm{yr}$ ago, during the previous RSG phase of the star. We note that the lifetime of unshielded $\mathrm{CO}$ against the average interstellar UV-field is only $\sim 100 \mathrm{yr}$ (van Dishoeck \& Black 1988). This implies that we are seeing only the densest inhomogeneities in the torus, where the $\mathrm{CO}$ is sufficiently shielded, which may explain the clumpy appearance.

This clumpy torus resembles the famous ring around the supernova SN 1987A, which is believed to have been ejected during the progenitor star's RSG phase (Smith et al. 2007). Several Galactic supergiants have also been found to have similar morphologies, with an equatorial ring and a bipolar hourglass nebula, e.g., SBW1 (Smith et al. 2013) and Sher 25 (Brandner et al. 1997). While IRAS 17163 does not appear to have an accompanying bipolar nebula, it is possible that it is no longer detectable in $\mathrm{CO}$ due to dissociation. There has also been some suggestion of an embedded disk or torus in the YHG IRC+10420 (Castro-Carrizo et al. 2007). The main proposed formation scenarios for the torus are either a fast, low-density wind expanding into a previous slow, dense wind with a strong equatorial density enhancement, or a burst of intense mass-loss from a rapidly rotating star. For a relatively large and cool star like IRAS 17163 , acquiring a sufficiently large rotation rate would probably require interaction or merger with a binary companion.

It is possible that the clumpy $\mathrm{CO}$ ring was not ejected by the star, but is instead swept up ISM material. The isotropic 
wind from the star could create a cavity in the ISM, pushing ISM clouds into a ring shape. However, the lack of an expanding spherical geometry would require the ISM to be significantly denser in a plane around the star before being swept up. Furthermore, the kinematic structure of the clumpy ring must be considered. The clumps show redshifted velocities to the southeast and blue-shifted velocities to the north-west, and there are clumps at the systemic velocity to the south-west and north-east. This might be explained by the star moving towards the southeast, pushing ISM ahead of it, though this is difficult to reconcile with the circular symmetry of the ring. Another aspect is the inter-clump velocity difference of $\sim 20 \mathrm{~km} \mathrm{~s}^{-1}$. It is possible that the swept-up ISM clouds could retain their relative velocities, resulting in the observed clumps at various velocities at the same distance from the star. However, typical ISM intercloud velocities are $\sim 5-10 \mathrm{~km} \mathrm{~s}^{-1}$ (see e.g. Malhotra 1994, and references therein), a factor of 2-4 lower than the observed value.

From the current data it is likely that the clumpy $\mathrm{CO}$ ring is a torus ejected by the star, the formation of which may suggest the existence of a binary companion. It is also possible that the clumpy CO ring is swept-up ISM material, but the maintenance of symmetry and a coherent kinematical structure between the various clumps is difficult to explain in this case. The kinematics also do not rule out the possibility of infalling material, but this is unlikely given the large scales involved: the $\mathrm{CO}$ ring is about $40^{\prime \prime} \approx 0.8 \mathrm{pc}$ across. Further observations, for example allowing for the determination of the ${ }^{12} \mathrm{C} /{ }^{13} \mathrm{C}$ ratio or the properties of the dust, might be able to elucidate the origin of this ring.

\subsection{Origin of the spur}

The spur is an unexpected feature, but we argue that it is associated with the star, for several reasons. First, it appears to spatially extend from the star to the clumpy $\mathrm{CO}$ ring, which would be an unlikely coincidence for an ISM cloud in the line of sight. Second, the velocity of the spur is offset by at least $\sim 60 \mathrm{~km} \mathrm{~s}^{-1}$ from Galactic rotation at any distance between 0 and $10 \mathrm{kpc}$ towards IRAS 17163. Third, a corresponding feature can be seen in the Herschel dust image from Hutsemékers et al. (2013), as shown in Fig. 4, in the form of an elongation in the direction of the $\mathrm{CO}$ spur. This suggests that the spur and clumpy $\mathrm{CO}$ ring have similar properties, including a dust temperature of $\sim 60 \mathrm{~K}$. Furthermore, the estimated mass of the spur and clumpy $\mathrm{CO}$ ring, 23-5 $M_{\odot}$, is roughly similar to the total gas mass estimated by Hutsemékers et al. (2013) of $\sim 7 M_{\odot}$. And finally, the ${ }^{12} \mathrm{CO} /{ }^{13} \mathrm{CO}$ ratio of $13_{-5}^{+10}$ is consistent with material processed by a RSG star. There is also some evidence of a unidirectional ejection in the YHG AFGL 2343 (Quintana-Lacaci et al. 2008).

To create a feature like the spur requires a break in symmetry. One potential explanation is that the star has undergone sustained, directed mass-loss on a timescale of a few $10^{3}$ yr (assuming an expansion velocity of $100 \mathrm{~km} \mathrm{~s}^{-1}$ ), although the mechanism is uncertain. O' Gorman et al. (2015) found a $2.5 \times 10^{-4} M_{\odot}$ dust feature offset by $400 \mathrm{AU}$ from the red supergiant VY CMa, and suggested a localised, long-lived magnetohydrodynamic disturbance could cause such directed mass-loss from the star. However, the formation timescale for this dust feature is only $\sim 20 \mathrm{yr}$, and such localised mass-loss would appear as random mass ejections on larger spatial scales. The spur of IRAS 17163 has an extent of $>8 \times 10^{4} \mathrm{AU}$, corresponding to a timescale of $>3700 \mathrm{yr}$ (assuming a velocity of $100 \mathrm{~km} \mathrm{~s}^{-1}$ ), so it is difficult to explain in the same way. The spur is also much more massive: $\sim 3 M_{\odot}$ (Sect. 3.5).
Is it possible that the spur is an interstellar cloud, with a large peculiar velocity and aligned with the star by chance? In this case, we can roughly estimate its linear size and hence its distance using Larson's Law (Larson 1981) of turbulence in molecular clouds: $\sigma_{v}=1.1 \times l^{0.38}$ where $\sigma_{v}$ is the velocity dispersion (linewidth) in $\mathrm{km} \mathrm{s}^{-1}$ and $l$ the cloud size in pc. Using a linewidth of $8 \mathrm{~km} \mathrm{~s}^{-1}$ this gives a huge linear size of $\sim 185 \mathrm{pc}$, and combined with the spur's angular size of $15^{\prime \prime}$ this implies a distance of $2500 \mathrm{kpc}$. Hence, the spur is very unlikely to be a molecular cloud.

\section{Conclusions}

Our knowledge of the mass-loss, evolution and surroundings of post-RSG stars is very limited. We report here observations of the circumstellar emission around the yellow hypergiant star IRAS 17163 with the ALMA Compact Array (ACA). In CO 21 , a spectrum taken at the star shows the broad faint plateau of a stellar wind, centered on $+18 \mathrm{~km} \mathrm{~s}^{-1}$. Hence we resolve the apparent velocity discrepancy previously found from $\mathrm{CO}$ and optical emission lines (Wallström et al. 2015). The stellar wind has a large expansion velocity of $100 \pm 10 \mathrm{~km} \mathrm{~s}^{-1}$ and a mass-loss rate of $8 \pm 1.5 \times 10^{-5} M_{\odot} \mathrm{yr}^{-1}$, under the assumption of a constant and isotropic wind. We also detect the $\mathrm{H} 30 \alpha$ recombination line of hydrogen towards the star. The line is centered on $21 \pm 3 \mathrm{~km} \mathrm{~s}^{-1}$ with a FWHM of $57 \pm 6 \mathrm{~km} \mathrm{~s}^{-1}$.

Our ACA CO observations reveal a complex circumstellar environment, contrasting with the symmetry of previous infrared images of the star. We detect the $\mathrm{CO}$ counterpart to the previously observed Herschel dust shell, in the form of a clumpy CO ring at $v_{\text {sys }} \pm 20 \mathrm{~km} \mathrm{~s}^{-1}$. However, this ring does not have the velocity structure of an expanding shell, rather it shows a strong velocity gradient with a position angle $\sim 100^{\circ}$. The structure resembles a torus, like the one famously seen around the supernova SN 1987A. The probable ejection scenarios for this torus suggests IRAS 17163 may have a binary companion. Further breaking the symmetry is an elongated spur, stretching between the central unresolved stellar wind and the $\mathrm{CO}$ ring, which peaks at a velocity around $+40 \mathrm{~km} \mathrm{~s}^{-1}$ with respect to the star. There is no obvious blueshifted counterpart to this feature on the other side of the star. The ${ }^{12} \mathrm{CO} /{ }^{13} \mathrm{CO}$ ratio in the spur is measured to be $13_{-5}^{+10}$, suggesting it was ejected by the star in its previous RSG phase.

These observations demonstrate the complexity of massive evolved stars. ALMA is now allowing us to study the morphology of their circumstellar envelopes, the gas kinematics, and their mass-loss prior to a supernova explosion.

Acknowledgements. We would like to thank the referee for their constructive comments which helped improve the quality and clarity of this manuscript. This paper makes use of the following ALMA data: ADS/ JAO.ALMA\#2013.1.00502.S. ALMA is a partnership of ESO (representing its member states), NSF (USA) and NINS (Japan), together with NRC (Canada) and NSC and ASIAA (Taiwan) and KASI (Republic of Korea), in cooperation with the Republic of Chile. The Joint ALMA Observatory is operated by ESO, AUI/NRAO and NAOJ. N.C. and G.Q.L. acknowledge that their research leading to these results has received funding from the European Research Council under the European Union's Seventh Framework Programme (FP/2007-2013) / ERC Grant Agreement No. 610256 (NANOCOSMOS). E.L. and R.Sz. were partially supported by the National Research Center, Poland, DEC-2013/08/M/ST9/00664, within the framework of the HECOLS International Associated Laboratory. A.Z. was supported by the Science and Technology Research Council through grant ST/L000768/1. H.O. acknowledges financial support from the Swedish Research Council. 


\section{References}

Arnal, E. M., Duronea, N. U., \& Testori, J. C. 2008, A\&A, 486, 807 Bernes, C. 1979, A\&A, 73, 67

Brandner, W., Grebel, E. K., Chu, Y.-H., \& Weis, K. 1997, ApJ, 475, L45

Castro-Carrizo, A., Quintana-Lacaci, G., Bujarrabal, V., Neri, R., \& Alcolea, J. 2007, A\&A, 465, 457

de Jager, C. 1998, A\&ARv, 8, 145

Dinh-V.-Trung, Muller, S., Lim, J., Kwok, S., \& Muthu, C. 2009, ApJ, 697, 409

Gonzalez, G., \& Wallerstein, G. 2000, AJ, 119, 1839

Hutsemékers, D., Cox, N. L. J., \& Vamvatira-Nakou, C. 2013, A\&A, 552, L6

Lagadec, E., Zijlstra, A. A., Oudmaijer, R. D., et al. 2011, A\&A, 534, L10

Larson, R. B. 1981, MNRAS, 194, 809

Malhotra, S. 1994, ApJ, 433, 687

O’Gorman, E., Vlemmings, W., Richards, A. M. S., et al. 2015, A\&A, 573, L1

Origlia, L., Oliva, E., Maiolino, R., et al. 2013, A\&A, 560, A46

Oudmaijer, R. D., Davies, B., de Wit, W.-J., \& Patel, M. 2009, in The Biggest, Baddest, Coolest Stars, eds. D. G. Luttermoser, B. J. Smith, \& R. E. Stencel, ASP Conf. Ser., 412, 17
Quintana-Lacaci, G., Bujarrabal, V., \& Castro-Carrizo, A. 2008, A\&A, 488, 203

Reid, M. J., Menten, K. M., Zheng, X. W., et al. 2009, ApJ, 700, 137

Schöier, F. L., \& Olofsson, H. 2001, A\&A, 368, 969

Smith, N., Bally, J., \& Walawender, J. 2007, AJ, 134, 846

Smith, N., Arnett, W. D., Bally, J., Ginsburg, A., \& Filippenko, A. V. 2013, MNRAS, 429, 1324

Szczerba, R., Siódmiak, N., Stasińska, G., \& Borkowski, J. 2007, A\&A, 469, 799

van der Tak, F. F. S., Black, J. H., Schöier, F. L., Jansen, D. J., \& van Dishoeck, E. F. 2007, A\&A, 468, 627

van Dishoeck, E. F., \& Black, J. H. 1988, ApJ, 334, 771

Vassilev, V., Meledin, D., Lapkin, I., et al. 2008, A\&A, 490, 1157

Wallström, S. H. J., Muller, S., Lagadec, E., et al. 2015, A\&A, 574, A139

Wilson, T. L., \& Rood, R. 1994, ARA\&A, 32, 191

Yang, B., Stancil, P. C., Balakrishnan, N., \& Forrey, R. C. 2010, ApJ, 718, 1062

Zijlstra, A. A., Chapman, J. M., te Lintel Hekkert, P., et al. 2001, MNRAS, 322, 280 
Appendix A: CO 2-1 channel map

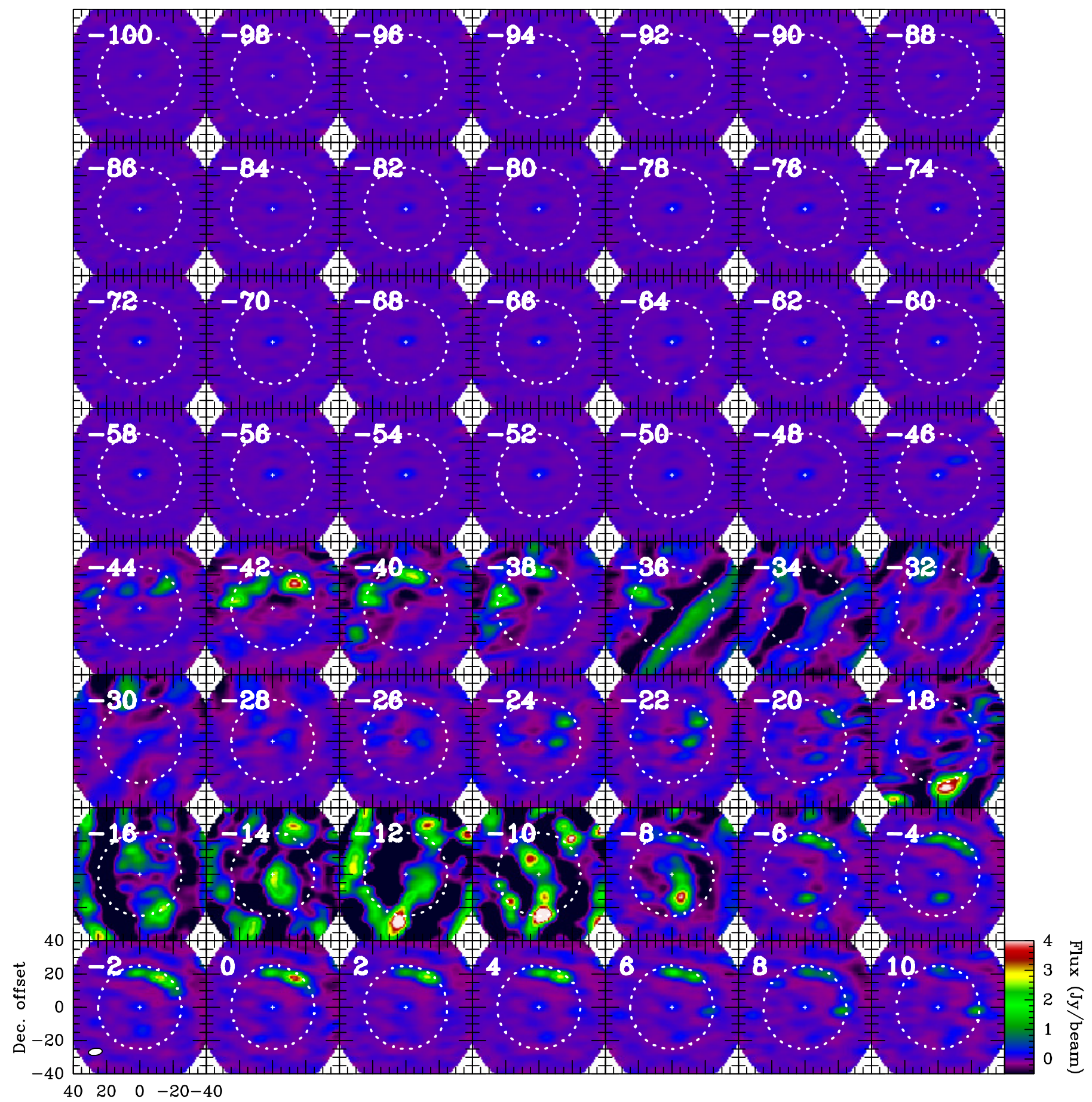

R.A. offset

Fig. A.1. ALMA ACA channel map of the CO 2-1 around IRAS 17163. The velocity of each channel is given in the top left; note the systemic velocity of the star is $+18 \mathrm{~km} \mathrm{~s}^{-1}$. The cool dust ring seen with Herschel is represented by a dashed circle with $25^{\prime \prime}$ radius, and the location of the star, at RA 17:19:49.33 Dec -39:10:37.94, is marked with a small cross. The restoring beam is shown as a white ellipse in the bottom left of the bottom left image. The common color scale is shown on the bottom right image. The figure continues on the next page. 


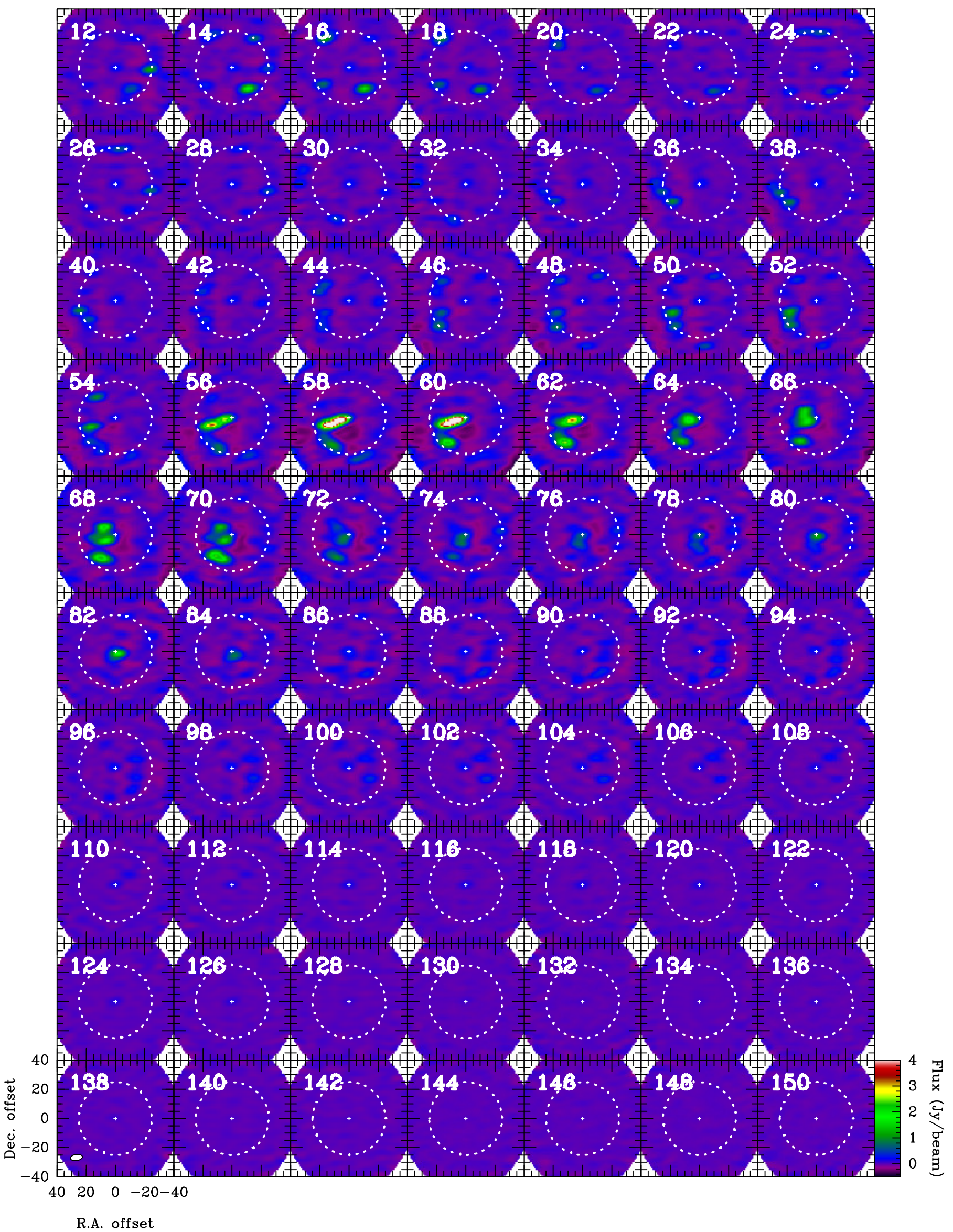

Fig. A.1. continued. 